\title{
Thioredoxin mitigates radiation-induced hematopoietic stem cell injury in mice
}

Pasupathi Sundaramoorthy ${ }^{1 \dagger}$, Qinhong Wang ${ }^{1 \dagger}$, Zhihong Zheng ${ }^{1,2}$, Yiqun Jiao ${ }^{1}$, Benny J. Chen ${ }^{1}$, Phuong L. Doan ${ }^{1}$, Nelson J. Chao ${ }^{1}$ and Yubin Kang ${ }^{1 *}$

\begin{abstract}
Background: Radiation exposure poses a significant threat to public health. Hematopoietic injury is one of the major manifestations of acute radiation sickness. Protection and/or mitigation of hematopoietic stem cells (HSCs) from radiation injury is an important goal in the development of medical countermeasure agents (MCM). We recently identified thioredoxin (TXN) as a novel molecule that has marked protective and proliferative effects on HSCs. In the current study, we investigated the effectiveness of TXN in rescuing mice from a lethal dose of total body radiation (TBI) and in enhancing hematopoietic reconstitution following a lethal dose of irradiation.
\end{abstract}

Methods: We used in-vivo and in-vitro methods to understand the biological and molecular mechanisms of TXN on radiation mitigation. BABL/C mice were used for the survival study and a flow cytometer was used to quantify the HSC population and cell senescence. A hematology analyzer was used for the peripheral blood cell count, including white blood cells (WBCs), red blood cells (RBCs), hemoglobin, and platelets. Colony forming unit (CFU) assay was used to study the colongenic function of HSCs. Hematoxylin and eosin staining was used to determine the bone marrow cellularity. Senescence-associated $\beta$-galactosidase assay was used for cell senescence. Western blot analysis was used to evaluate the DNA damage and senescence protein expression. Immunofluorescence staining was used to measure the expression of $\mathrm{Y}-\mathrm{H} 2 \mathrm{AX}$ foci for DNA damage.

Results: We found that administration of TXN 24 h following irradiation significantly mitigates BALB/c mice from TBI-induced death: $70 \%$ of TXN-treated mice survived, whereas only $25 \%$ of saline-treated mice survived. TXN administration led to enhanced recovery of peripheral blood cell counts, bone marrow cellularity, and HSC population as measured by c-Kit ${ }^{+}$Sca- $1^{+}$Lin $^{-}$(KSL) cells, SLAM + KSL cells and CFUs. TXN treatment reduced cell senescence and radiation-induced double-strand DNA breaks in both murine bone marrow lineage-negative (Lin ${ }^{-}$) cells and primary fibroblasts. Furthermore, TXN decreased the expression of p16 and phosphorylated p38. Our data suggest that TXN modulates diverse cellular processes of HSCs.

Conclusions: Administration of TXN $24 \mathrm{~h}$ following irradiation mitigates radiation-induced lethality. To the best of our knowledge, this is the first report demonstrating that TXN reduces radiation-induced lethality. TXN shows potential utility in the mitigation of radiation-induced hematopoietic injury.

Keywords: Thioredoxin, Radiation exposure, Mitigation, Hematopoietic stem cells, Senescence

\footnotetext{
*Correspondence: yubin.kang@duke.edu

'Equal contributors

'Division of Hematological Malignancies and Cellular Therapy, Duke

University Medical Center, DUMC 3961, 2400 Pratt Street, Suite 9000,

Durham, NC 27710, USA

Full list of author information is available at the end of the article
} 


\section{Background}

Radiation and radioactive substances are used extensively in medical research, disease diagnosis, and cancer treatment. Incidental radiation exposure could result from medical and radiological accidents, malfunction or breakdown of nuclear power plants, or a terrorist attack with radioactive dirty bombs. The danger of radiation injury is real, particularly in the aftermath of the $9 / 11$ attacks in the US. Radiation injury impacts public health and society significantly. Between 1945 and 1987, there were 285 nuclear reactor accidents, injuring 1550 people and killing 64 [1-5]. These nuclear and radiological emergencies require comprehensive medical preparedness and readiness, including a national stockpile of deliverable agents to counteract radiation exposure incidents and accidents.

Hematopoietic stem cells (HSCs) and hematopoiesis are among the tissues/organs most sensitive to radiation injury and contribute to many of the manifestations of acute radiation injury, including bleeding, infection, and bone marrow failure. Evidence has emerged that excessive reactive oxygen species (ROS) following radiation injury cause HSC apoptosis and senescence and the loss of long-term repopulating capacity [6-9]. However, what remains poorly understood is how to harness the redox pathway for the mitigation of radiation-induced HSC injury. N-acetyl-L-cysteine (NAC) and glutathione have been shown to be able to protect HSCs from oxidative stress and promote the recovery of hematopoiesis when administered before radiation or immediately following radiation exposure. However, these compounds need to be given frequently (at least daily) and in large quantities (at least $100 \mathrm{mg} / \mathrm{kg}$ in mice) [10, 11]. Hematopoietic growth factors (HGFs) such as erythropoietin, thrombopoietin, and granulocyte-colony stimulating factor (G-CSF) have been tested in radiation injury for enhancing hematopoietic recovery [12, 13]. However, HGFs have limitations and drawbacks; these factors are lineage-specific and therefore do not promote the recovery of other cell lineages. Additionally, the efficacy of these growth factors is limited; G-CSF only quickens neutrophil recovery by a few days, and works only with a low dose of radiation exposure [11, 14-16]. Thrombopoietin needs to be administered soon after radiation exposure, and its efficacy is significantly limited if given $24 \mathrm{~h}$ after radiation exposure [17]. Currently, there is an urgent need to identify and develop novel agents that can be used to reduce radiation-induced HSC injury and enhance all lineage hematopoietic recovery when given $24 \mathrm{~h}$ after radiation exposure.

Using a semiquantitative, mass spectrometry-based proteomic approach, we recently screened for proteins that were differentially expressed in the bone marrow supernatants from hematopoietic stem cell transplant recipient mice that were treated with AMD3100 (a specific and reversible CXCR4 antagonist) [18]. We identified thioredoxin (TXN) as a novel molecule that has marked protective and proliferative effects on HSCs. TXN is a ubiquitous oxidoreductase with a molecular weight of $12 \mathrm{kDa}$ and has two Cys residues in the conserved active site sequence (-Cys32-Gly-Pro-Cys35-). The primary function of TXN is to maintain redox homeostasis and to protect proteins from oxidative damage or inactivation [19-21]. We have shown that exvivo culture of murine HSCs with TXN or giving TXN to HSC transplant recipient mice enhanced the recovery and the long-term repopulation capacity of HSCs in our mouse models of HSC transplant [18]. However, the therapeutic potential of TXN as a radiation protectant or mitigator has not been investigated.

In the current study, we aimed to determine the effects of TXN in mitigating radiation-induced HSC injury when given $24 \mathrm{~h}$ after radiation exposure. Twenty-four hours after radiation has been chosen as a critical time point for evaluating the efficacy of any given agent as a radiation mitigator because, in the event of a radiation mass casualty scenario, a significant majority of patients will not present for therapeutic intervention for several hours following radiation exposure. To the best of our knowledge, this is the first report showing TXN to be an important agent for rescuing radiationinduced hematopoietic injury.

\section{Methods}

Mice

Eight- to 12-week-old female BALB/c mice were purchased from Jackson Laboratories (Bar Harbor, ME, USA) and used in this study. The mice were housed in our specific pathogen-free facility and maintained at $23-25^{\circ} \mathrm{C}$ with a $12 \mathrm{~h}$ day/ $12 \mathrm{~h}$ dark cycle throughout the study, and were provided with autoclaved food and acidified water. All our studies were performed in accordance with Duke University Institutional Animal Care and Use Committee approved procedures.

\section{Cell culture}

All the cell lines were purchased from American Type Culture Collection (Manassa, VA, USA). Primary fibroblast cells were grown in RPMI 1640 medium. The medium was supplemented with $10 \%$ fetal bovine serum (FBS) and $1 \%$ penicillin/streptomycin. Cells were maintained at $37{ }^{\circ} \mathrm{C}$ with $5 \% \mathrm{CO}_{2}$ in a humidified incubator.

Murine lineage-negative $\left(\mathrm{Lin}^{-}\right)$cells were isolated from bone marrow cells (BMCs) using a lineage negative selection column as per the manufacturer's instruction (Miltenyi Biotec Inc., Auburn, CA, USA). Briefly, BMCs were harvested from bilateral femurs and tibias of $\mathrm{BALB} / \mathrm{c}$ mice and depleted of red blood cells (RBCs) 
using ACK lysis buffer (Lonza, Walkersville, MD, USA). $\mathrm{Lin}^{-}$cells were then enriched using lineage negative selection columns. Isolated $\mathrm{Lin}^{-}$cells were grown with $20 \mathrm{ng} / \mathrm{ml}$ mouse thrombopoietin (TPO), $125 \mathrm{ng} /$ $\mathrm{ml}$ mouse stem cell factor (SCF), and $50 \mathrm{ng} / \mathrm{ml}$ mouse Flt3 ligand in 10\% FBS with IMDM medium. Cells were maintained at $37{ }^{\circ} \mathrm{C}$ with $5 \% \mathrm{CO}_{2}$ in a humidified incubator.

\section{Reagents and antibodies}

Recombinant human thioredoxin-1 (TXN; E. coli-derived) was purchased from R\&D Systems Inc. (Minneapolis, MN, USA). PE-conjugated anti-mouse Scal-1 and PerCP5.5 lineage cocktail antibody was obtained from BD Biosciences (San Jose, CA, USA). FITC-conjugated antimouse CD48 and APC-conjugated anti-mouse CD 150 were obtained from Biolegend (San Diego, CA, USA). PECyanine 7-conjugated anti-mouse CD117 (c-Kit) was purchased from eBioscience Inc. (San Diego, CA, USA). Senescence $\beta$-galactosidase (SA-gal) staining kit, $\gamma$-H2AX, p38 mitogen-activated protein kinase (MAPK), phosphop38 MAPK (Thr180/Tyr182), p16, $\beta$-actin, and the corresponding secondary antibodies were purchased from Cell Signaling Technology (Danvers, Massachusetts, USA).

\section{Radiation injury mouse model and TXN treatment}

BALB/c mice were irradiated with 7.25 Gy by total body irradiation (TBI) using a ${ }^{137}$ Cesium gamma irradiator (JL Shepherd, Glendale, CA, USA) at a dose rate of $4.18 \mathrm{~Gy} / \mathrm{min}$ [22]. Mice were irradiated on a rotating platform. Twenty-four hours later, the mice were injected via the tail vein with TXN $(1.6 \mathrm{mg} / \mathrm{kg})$ every other day for a total of five doses, and the control group was administered with phosphate-buffered saline (PBS). Animal survival was monitored daily up to 30 days. In a separate sets of experiments, blood samples and BMCs were collected at 3 weeks or 6 weeks after radiation from surviving mice. Peripheral blood cell counts were measured by hematology analyzer. BMCs from femurs and tibias of the mice were measured for HSC population and cell senescence by flow cytometry and colony forming units (CFU).

\section{Peripheral blood analysis}

Blood was directly withdrawn from the maxillary vein and collected in pre-coded EDTA-containing vials at 3 and 6 weeks following radiation exposure from surviving mice. Blood was mixed gently on a rotary shaker until analysis for white blood cells (WBCs), hemoglobin, and platelets on a hematology analyzer (scil Vet ABC Plus ${ }^{\mathrm{Tw}}$, Gurnee, IL, USA).

\section{Flow cytometry analysis}

To determine the percentage and absolute number of c-Kit ${ }^{+} \mathrm{Sca}-1^{+} \mathrm{Lin}^{-}$(KSL) and SLAM + KSL cells after radiation from surviving mice, the mice were sacrificed and bones ( 2 femurs and 2 tibias per mouse) were harvested as described previously [18]. The cells were stained with PE-Cy7-CD117 (c-Kit), PE-Sca-1, and PerCP-5.5 lineage antibody, FITC-CD48, and APCCD150 for $30 \mathrm{~min}$ for KSL and SLAM + KSL cell percentage. Acquisition was carried out using a BDCanto II flow cytometer with the FACSDiva software (Becton Dickinson, San Jose, CA, USA).

\section{CFU assay}

CFU assays were performed in complete M3434 methylcellulose medium (Stem Cell Technologies, USA) following the manufacturer's instructions. Briefly, BMCs were mixed in complete M3434 medium and plated in $30-\mathrm{mm}$ petri dishes at 20,000 cells per dish. The assay was performed in triplicate and the number of colony forming units-granulocyte macrophage (CFU-GM) and colony forming units-granulocyte, erythroid, macrophage, megakaryocyte (CFU-GEMM) were counted at day 7 and day 12, respectively.

\section{Bone marrow histology examination}

Femurs were fixed in 10\% neutral-buffered formalin. Specimens were then decalcified, embedded in paraffin, cut into $5-\mu \mathrm{m}$ section, and stained with hematoxylin and eosin (H\&E). The slides were examined by light microscopy to capture bright-field images using an Olympus (IX51) microscope (Japan).

\section{SA-gal activity analysis}

To determine $\beta$-gal + senescent bone marrow cells, bone marrow was harvested and $\mathrm{Lin}^{-}$cells were isolated by magnetic column purification using a mouse lineage cell depletion kit (Miltenyi Biotec) as described previously [18]. SA-gal activity in $\mathrm{Lin}^{-}$cells was determined using a SA-gal staining kit from Cell Signaling Technology (Beverly, MA, USA) according to the manufacturer's instructions.

Primary fibroblast cells were seeded into six-well plates at $1 \times 10^{4}$ cells per well and incubated in a humidified atmosphere with $5 \% \mathrm{CO}_{2}$ at $37{ }^{\circ} \mathrm{C}$ overnight. Cells were then irradiated with $3 \mathrm{~Gy}$ or $5 \mathrm{~Gy}$ and treated with TXN $10 \mu \mathrm{g} / \mathrm{ml}$. Three days after irradiation, the cells were fixed and stained with the SA-gal staining kit as per the manufacturer's instructions.

\section{Western blot}

Cells were harvested, washed with PBS, and re-suspended in lysis buffer containing $50 \mathrm{mM}$ Tris- $\mathrm{HCl} \mathrm{pH}$ 7.4, $150 \mathrm{mM} \mathrm{NaCl}, 1 \mathrm{mM}$ EDTA, $1 \%$ Triton $\times 100,1 \%$ sodium 
deoxycholate, and $0.1 \%$ SDS. The cells were further lysed by brief sonication. The lysates were centrifuged at high speed for $10 \mathrm{~min}$ to remove the cell debris. Total protein was quantified using the DC protein estimation kit (Bio Rad) with bovine serum albumin (BSA) as a standard. Approximately $20 \mu \mathrm{g}$ protein was loaded and run on SDSPAGE. The proteins were transferred onto a nitrocellulose membrane. The membrane was blocked with $5 \%$ milk in Tris-buffered saline containing $0.1 \%$ Tween 20 (TBST), and primary antibodies were applied with 5\% BSA in TBST overnight at $4{ }^{\circ} \mathrm{C}$ with gentle shaking. The membrane was then probed with HRP-conjugated secondary antibody and developed using the Pierce ECL substrate.

\section{Immunofluorescence}

Primary fibroblast cells were irradiated (3 Gy) and cultured with and without TXN for $24 \mathrm{~h}$. Cells were fixed in $100 \%$ cold ethanol for $10 \mathrm{~min}$ at $-20^{\circ} \mathrm{C}$, permeabilized with Triton $\times 100$ and washed with PBS. After blocking, cells were incubated overnight at $4{ }^{\circ} \mathrm{C}$ with anti- $\gamma \mathrm{H} 2 \mathrm{AX}$ rabbit antibody. The excess of unbound antibody was removed at each step by three washes with PBS. The cells were counterstained with DAPI. The images were captured on an Olympus confocal microscope (FV1000MPE, Olympus, Tokyo, Japan).

\section{Statistical analysis}

All the data are presented as the mean \pm SD. Comparisons were performed either by the student $t$ test for analysis of variance for continuous data or by log-rank test for survival data. All statistical analyses were performed using Star View software (SAS institute, Cary, NC, USA) or Microsoft Excel (Microsoft, Seattle, WA, USA). P values less than 0.05 were considered significant.

\section{Results}

TXN rescues mice from a lethal dose of total body irradiation even when administered $24 \mathrm{~h}$ after irradiation TXN has two major functions. First, TXN serves as one of the major antioxidants in mammals and protects cells from oxidative stress. Second, TXN is a cell growth factor and can modulate and stimulate diverse cellular processes by directly interacting with redox-sensitive or ROS-independent molecular pathways $[20,21]$. TXN is an excellent candidate for drug development because of its structural stability, its ability to cross the cell membrane, and its ubiquitous expression. Previously, we found that TXN protected C57BL/6 mice from radiation-induced hematological injury and death when given $2 \mathrm{~h}$ after radiation exposure [18]. To test whether the protective effect of recombinant TXN can be generalized to other strains of mice and if TXN is still
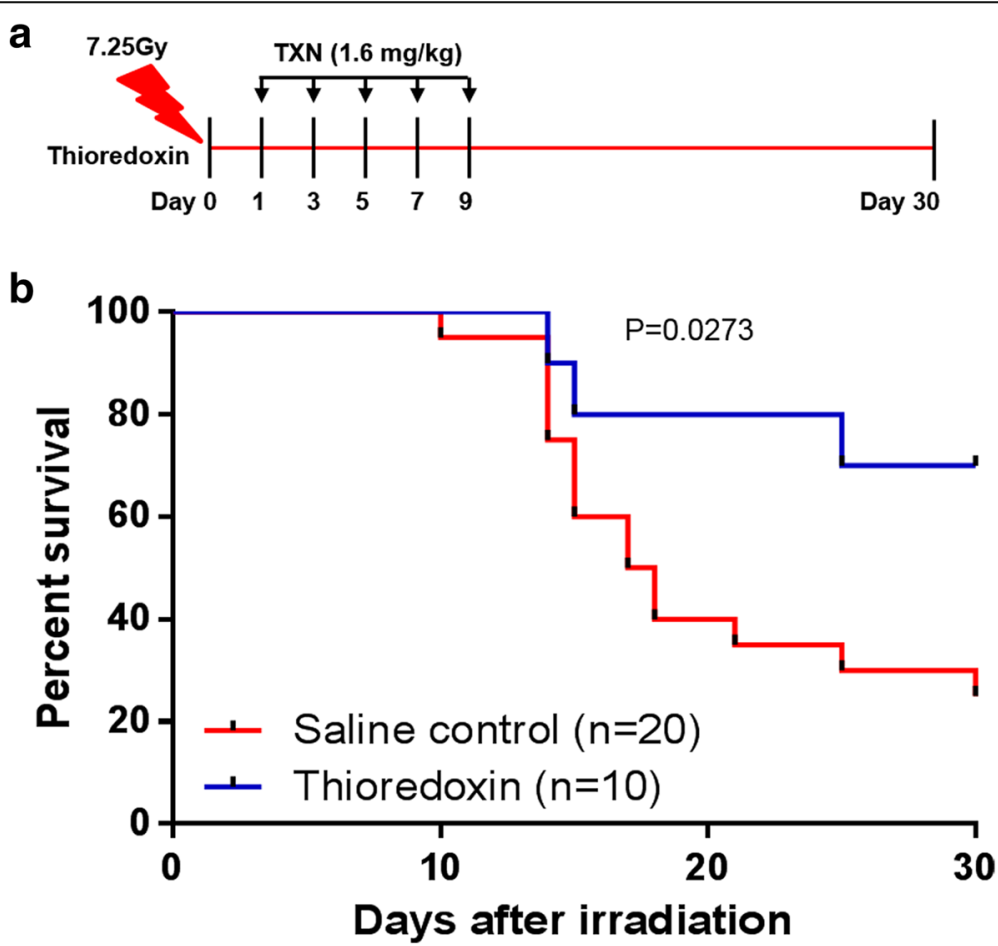

Fig. 1 TXN mitigates TBI-induced lethality in mice. a Radiation and thioredoxin (TXN) treatment scheme for mice. BABL/c mice were irradiated with $7.25 \mathrm{~Gy}$, and $1.6 \mathrm{mg} / \mathrm{kg}$ of TXN was administrated intravenously by tail vein at $24 \mathrm{~h}$ after irradiation and then every other day for a total of five doses. $\mathbf{b}$ The protective effects of TXN. Kaplan-Meier survival curve depicts the 30 day survival $(n=20$ for saline-treated, lethally irradiated mice, and $n=10$ for TXN-treated, lethally irradiated mice). One of two separate sets of experiments is shown 
effective when given at $24 \mathrm{~h}$ after irradiation, BALB/c mice were total body irradiated with 7.25 Gy. Twentyfour hours later, the mice were given intravenous PBS control buffer or TXN at $32 \mu \mathrm{g}$ per mouse $(1.6 \mathrm{mg} / \mathrm{kg}$ body weight). The treatment was continued every other day for a total of five doses (Fig. 1a). The mouse survival was observed for 30 days. As shown in Fig. 1b, Kaplan-Meier analysis of survival indicated that TXN rescued mice from a lethal dose of radiation: $70 \%$ of TXN treated-mice survived the radiation whereas only $25 \%$ of saline-treated mice survived $(p=0.0273)$. These results suggested that TXN mitigates a lethal dose of TBI even when administered $24 \mathrm{~h}$ after radiation exposure.

TXN enhances the reconstitution of murine hematopoietic stem cells after a lethal dose of total body irradiation

Radiation induces HSC damage and affects all lineages of blood cells. Protecting and rescuing HSCs from radiation damage are important in the treatment of radiation injury $[8,23]$. Therefore, we sought to investigate the in-vivo effect of treatment with TXN on hematopoietic cells. BALB/c mice were irradiated with 7.25 Gy followed by TXN administration $24 \mathrm{~h}$ later as described in Fig. 1a. Peripheral blood cell counts were measured at 3 weeks and 6 weeks after radiation in surviving mice. As shown in Fig. 2a, WBC counts were significantly increased in TXN-treated mice at 3 weeks following radiation in comparison to saline-treated mice $(p<0.05)$. The levels of RBCs and hemoglobin were not significantly changed by TXN treatment, although there was a trend for higher numbers of RBCs and hemoglobin in TXN-treated mice at 3 weeks following radiation (Fig. $2 \mathrm{~b}$ and $\mathrm{c}$ ). The platelet levels were significantly increased at both 3 and 6 weeks $(p<0.05)$ by TXN administration (Fig. 2d). These data indicate a faster hematological recovery of WBCs and platelets following TXN treatment after radiation exposure.

We next analyzed the bone marrow HSC population at 3 weeks and 6 weeks after radiation. We measured the percentage and the absolute number per femur of bone marrow KSL cells and $\mathrm{SLAM}^{+} \mathrm{KSL}$ cells using FACS analysis. KSL cells are a mixed population of murine hematopoietic stem cells and hematopoietic progenitor cells. SLAM ${ }^{+}$KSL cells represent primitive, long-term repopulating hematopoietic stem cells [24]. We found that TXN given at $24 \mathrm{~h}$ after irradiation significantly increased the numbers of KSL cells (Fig. 3a) $(p<0.05)$ and SLAM ${ }^{+}$KSL cells (Fig. 3b) $(p<0.05)$.

The numbers of colony forming units (CFUs) serve as an indicator for hematopoiesis and an important sign for hematopoietic recovery. We thus measured bone marrow CFUs in TXN-treated mice and saline-treated mice
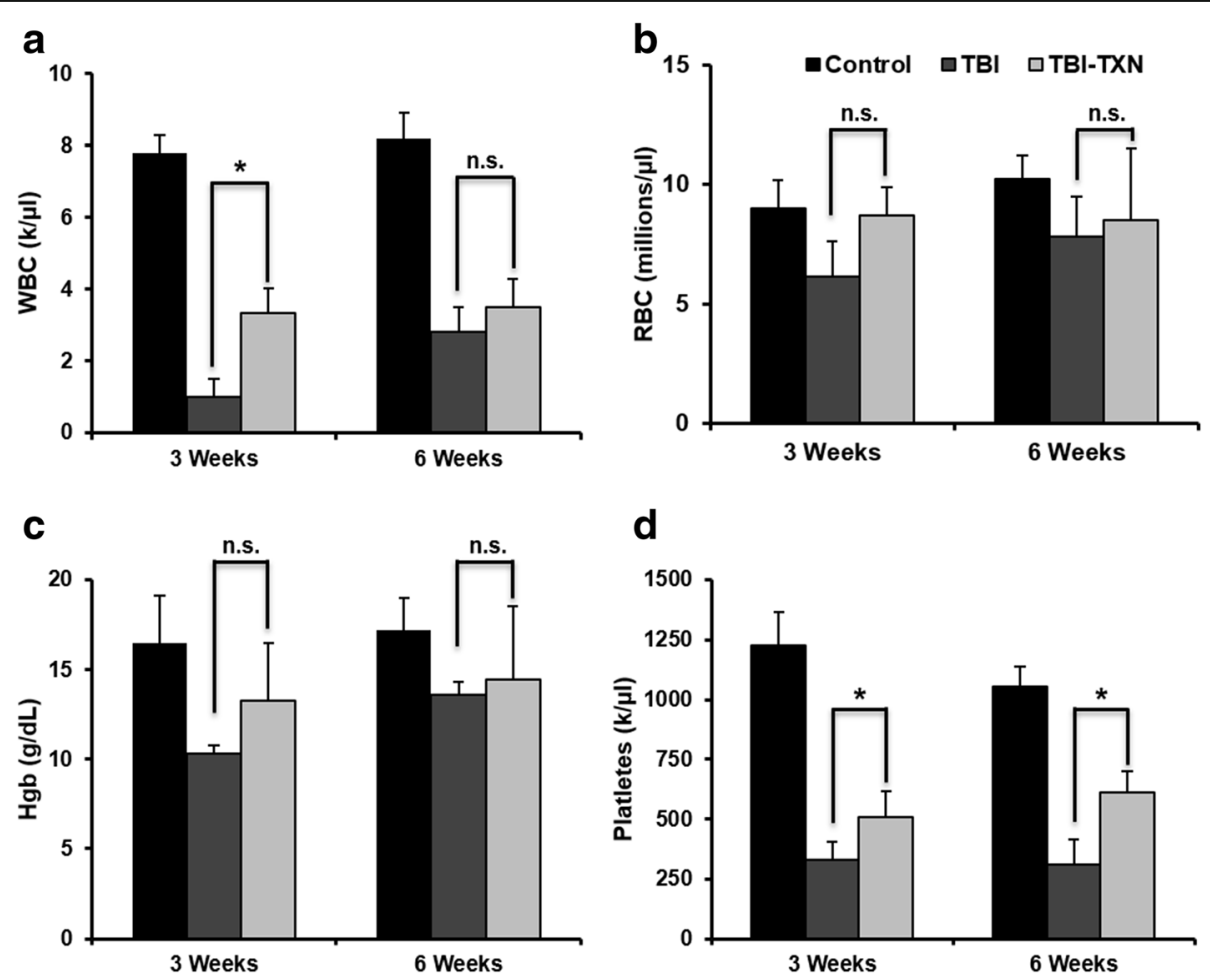

Fig. 2 TXN mitigates TBI-induced pancytopenia in mice. Mice were irradiated with 7.25 Gy and then treated with saline or thioredoxin (TXM) as described in the text. The numbers of a white blood cells $(W B C)$, b red blood cells (RBC), $\mathbf{c}$ hemoglobin (Hgb), and $\mathbf{d}$ platelets in peripheral blood were quantified at 3 and 6 weeks after total body irradiation (TB). Data are shown as the mean \pm SD. ${ }^{*} P<0.05$. n.s. not significant 

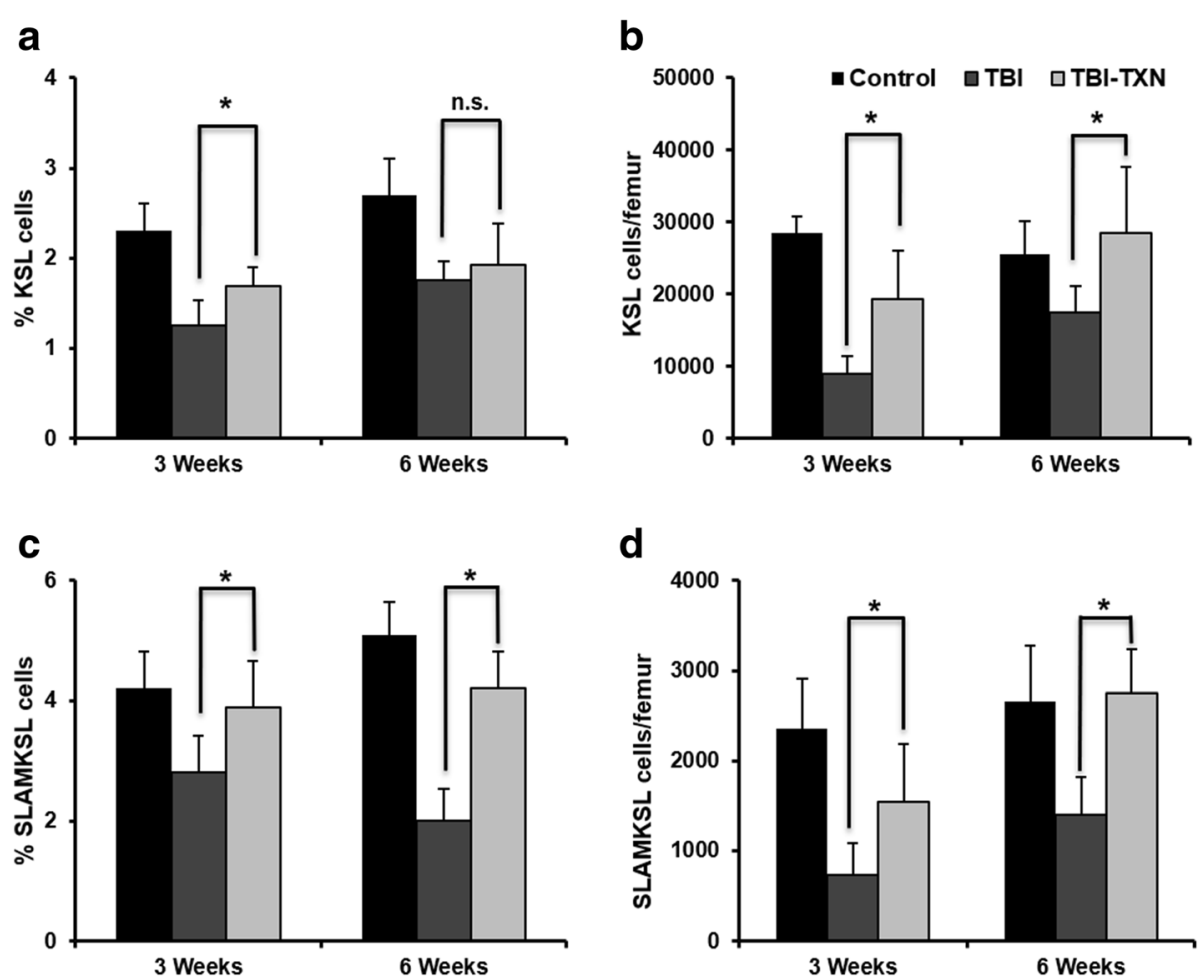

Fig. 3 TXN mitigates TBI-induced suppression of HSCs in mice. Mice were irradiated with $7.25 \mathrm{~Gy}$ and then treated with saline or thioredoxin (TXN) as described in the text. The frequency of HSCs in bone marrow was analyzed by flow cytometry; a percentage of KSL cells and $\mathbf{b}$ the absolute number of KSL cells/femur; c percentage of SLAM + KSL (SLAMKSL) and $\mathbf{d}$ the absolute number of SLAM + KSL cells/femur. Data are shown as the mean \pm SD. ${ }^{*} P<0.05 . K^{K S L}$ c-Kit ${ }^{+}$Sca- $1^{+}$Lin $^{-}$, n.S. not significant, $T B /$ total body irradiation

at 3 weeks and 6 weeks after lethal irradiation. We found that TXN administration significantly increased CFUGM and CFU-GEMM (Fig. 4a and b) $(p<0.05)$. Radiation-induced bone marrow damage resulted in massive ablation of the cellular content in the bone marrow, and decreased bone marrow nucleated cells (BMNCs) [23]. We thus examined the bone marrow cellular content by H\&E staining and found that TXN treatment reduced the radiation-induced cellular depletion and increased bone marrow cellularity (Fig. 4c). These data suggest that TXN mitigates the TBI-induced bone marrow damage by enhancing hematopoiesis and facilitating stem cell regeneration to accelerate the hematopoietic recovery.

TXN reduces cell senescence after a lethal dose of TBI

Cell senescence occurs after exposure to radiation and is one of the major biological processes that leads to the impairment of HSC function and the loss of HSC selfrenewal capacity $[7,25]$. Therefore, we examined the effects of TXN on radiation-induced cell senescence in vitro and in vivo. BALB/c mice were irradiated and treated with TXN or saline as described in Fig. 1a. At 3 weeks after radiation, bone marrow Lin $^{-}$cells were isolated from the mice and stained for senescence- associated $\beta$-galactosidase (SA-gal) activities. TXN significantly reduced radiation-induced cell senescence in murine bone marrow Lin $^{-}$cells by FACS analysis (Fig. 5a and b).

To further confirm the effects of TXN in mitigating radiation-induced cell senescence, we evaluated the TXN effect in vitro using primary fibroblasts. We irradiated primary fibroblasts with 3 Gy or 5 Gy and cultured the cells with or without TXN for $72 \mathrm{~h}$. We chose $3 \mathrm{~Gy}$ and 5 Gy because 3 Gy was found to cause hematopoietic damage symptoms [1] and 5 Gy could result in death in $50 \%$ of exposed individuals from the sequelae of hematopoietic damage unless there is medical intervention [2]. As shown in Fig. $5 \mathrm{c}$ and d, irradiation induced cell senescence as demonstrated by increased SA-gal-positive cells after irradiation. TXN treatment significantly reduced SA-gal-positive primary fibroblasts, indicating that TXN treatment reduced radiation-induced cell senescence in primary fibroblasts.

p16 (cyclin-dependent kinase inhibitor 2A) has been involved in the establishment and maintenance of cellular senescence and is an important marker of cellular senescence $[25,26]$. Consistent with SA-gal activity, the expression of p16 level was reduced with TXN treatment (Fig. 5e and f). Collectively, these 

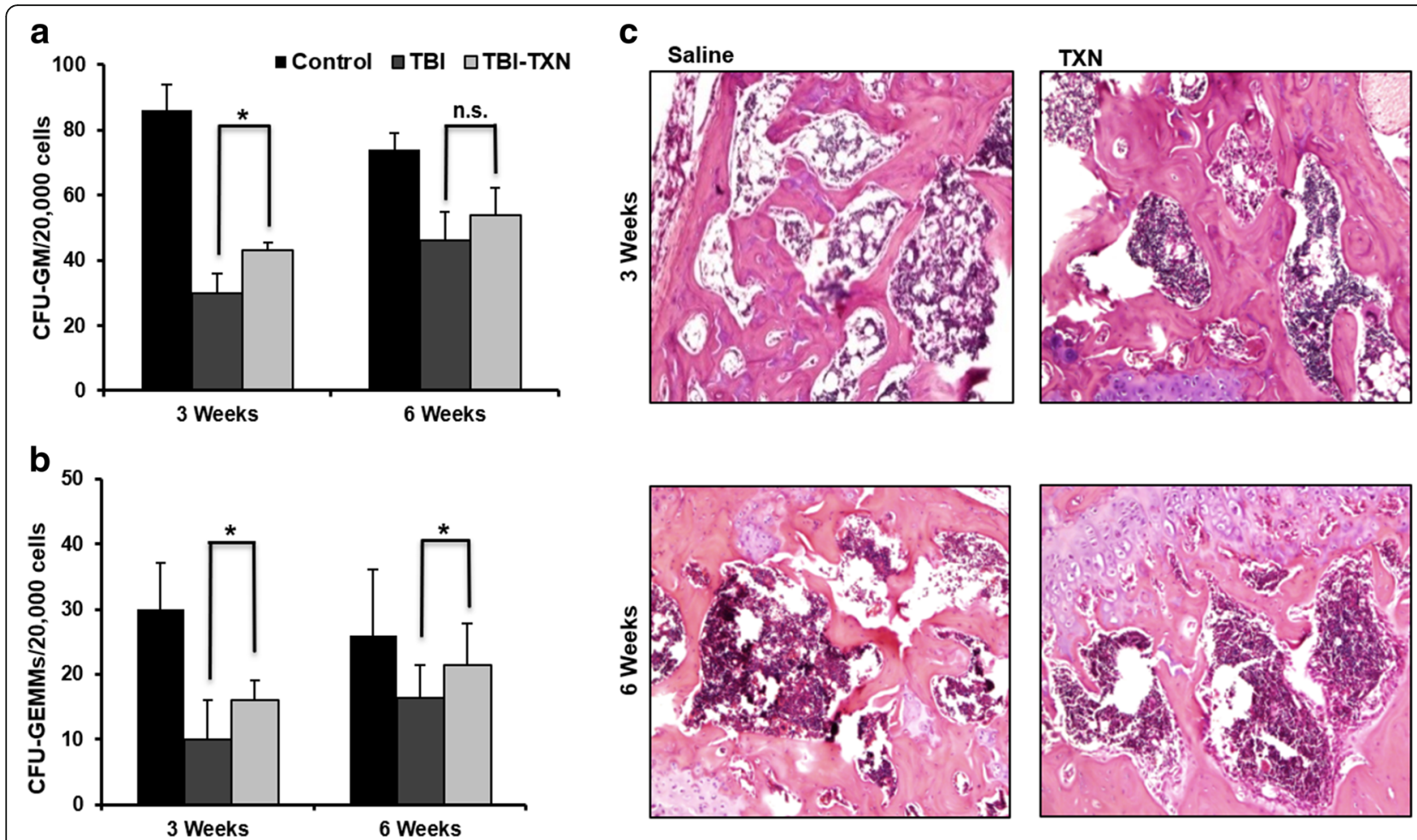

Fig. 4 TXN facilitates expansion of hematopoietic progenitor cells and increases bone marrow cellularity. The effects of thioredoxin (TXN) on the colongenic function of HSCs in bone marrow were measured by CFU assay in total body irradiation (TBI) mice for a colony forming unit-granulocyte macrophage (CFU-GM) and $\mathbf{b}$ colony forming unit-granulocyte, erythroid, macrophage, megakaryocyte (CFU-GEMM). $\mathbf{c}$ Effects of TXN on bone marrow cellularity in TBI mice. Panels show H\&E staining of mouse femurs. Representative images are shown for saline and TXN treatments. Data are shown as the mean $\pm S D$. ${ }^{*} P<0.05$. n.s. not significant

data suggest that TXN treatment rescues cells from radiation-induced cell senescence.

Studies have found that p38 MAPK is activated after radiation exposure and the activated p38 mediates cell senescence, apoptosis, and loss of self-renewal capacity in HSCs [27-29]. To determine the effects of TXN on p38 in murine hematopoietic cells, murine bone marrow cells were enriched for hematopoietic stem/progenitor cells using a lineage-deletion column. Murine Lin $^{-}$cells were irradiated and treated with TXN. Phosphorylated p38 (Thr180/Tyr182) and total p38 levels were measured at 1 and $4 \mathrm{~h}$ after irradiation. TXN treatment downregulated radiation-induced phosphorylated-p38 (Thr180/Tyr182) in murine $\mathrm{Lin}^{-}$bone marrow cells (Fig. 6a and b).

TXN reduces radiation-induced DNA double-strand breaks DNA double-strand break is a hallmark of radiation damage. Histone $\mathrm{H} 2 \mathrm{AX}$ becomes phosphorylated on serine 139, called $\gamma-\mathrm{H} 2 \mathrm{AX}$, as a reaction to DNA double-strand breaks. $\gamma$-H2AX has been used as a marker for DNA double-strand breaks [30, 31]. To test if TXN could reduce DNA double-strand breaks and/or enhance DNA repair, we measured the effects of TXN on $\gamma$-H2AX. Murine $\mathrm{Lin}^{-} \mathrm{BMCs}$ were irradiated with 3 Gy and treated with and without TXN for 1 and $4 \mathrm{~h}$. We found that TXN treatment led to a reduced level of $\gamma$-H2AX expression after radiation (Fig. 6a and c). Additionally, we measured $\gamma-\mathrm{H} 2 \mathrm{AX}$ foci in primary fibroblast cells (Fig. 6d). Consistent with the results in murine Lin $^{-}$cells, TXN significantly reduced the level of $\gamma$-H2AX in primary fibroblasts.

\section{Discussion}

Accidental radiation exposure or a terrorist attack with a radioactive dirty bomb poses a serious threat to public health. Management of radiation injuries is a complex medical challenge, requiring a careful encounter as well as therapeutic agents administered at the appropriate time following the radiation exposure [1, 23, 32]. HSCs and hematopoiesis are among the tissues/organs most vulnerable to radiation injury. Radiation-induced damage to HSCs leads to HSC cell senescence and defects in HSC self-renewal capacity, and contributes to several manifestations of acute radiation sickness. Currently, there are very few agents that can effectively rescue HSCs from radiation injury when given after radiation exposure [10]. Antioxidant and HSC growth factors have 


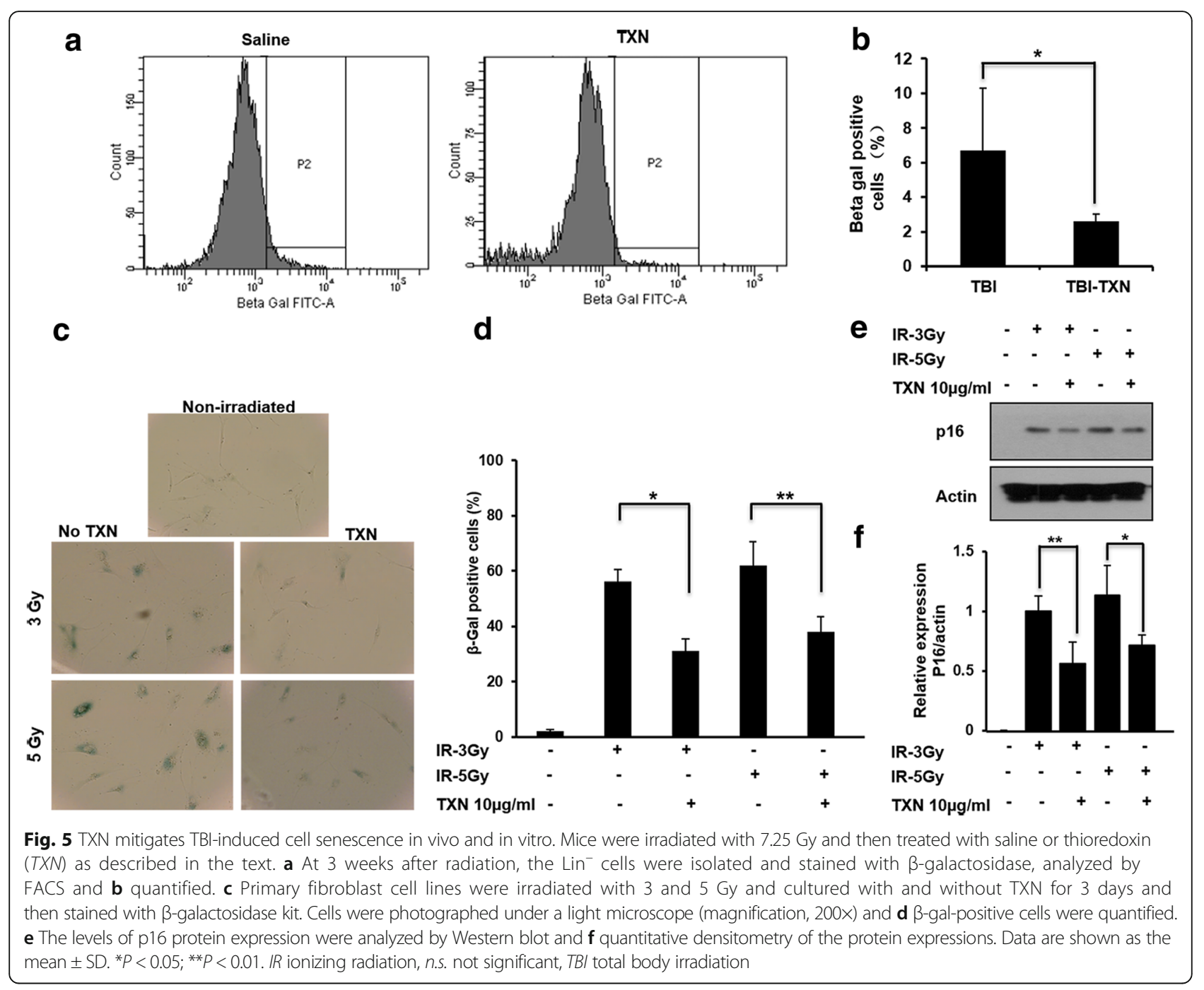

limitations, and the major drawback of these agents is that they should be administrated before radiation or immediately after radiation [33-35]. There is an unmet medical need for identifying and developing effective agents that can be used to rescue lethal-dose radiation injury and enhance all-lineage hematopoietic cell recovery when given after irradiation.

Previously, we reported that TXN mitigates mice from radiation-induced death and enhances HSC recovery when given at $2 \mathrm{~h}$ after radiation exposure in a mouse model of radiation injury [18]. Our current study has important clinical relevance. Individuals exposed to radiation may not be aware of the exposure until a few hours later. Therefore, agents that are effective in rescuing victims from radiation injury when given $24 \mathrm{~h}$ after radiation exposure have great potential for clinical use. The remarkable ability of TXN to mitigate radiationinduced damage when administered intravenously $24 \mathrm{~h}$ after lethal TBI makes it an attractive radiation countermeasure agent for further development in the use against radiation injury. Further supporting this proposition is that TXN is highly competent in ameliorating the radiation-induced hematopoietic injury by facilitating HSC recovery. We have shown that TXN enhanced the recovery of multi-lineages of peripheral blood cells such as WBCs and platelets. TXN-treated mice demonstrated more cellular bone marrow. Importantly, TXN treatment had a higher number of CFUGEMM, KSL cells, and primitive SLAM ${ }^{+}$KSL HSCs.

Radiation causes HSC damage through several mechanisms: increased production of ROS and induction of oxidative stress [36, 37]; increased oxidative DNA damage [38, 39]; activation of apoptotic cell death [40]; enhanced cell senescence [41, 42]; and promotion of HSC differentiation [43]. Cell senescence, an irreversible proliferative arrest, plays a critical role in radiation-induced HSC injury. HSC senescence impairs HSC replication and self-renewal, and thus reduces the HSC long-term 


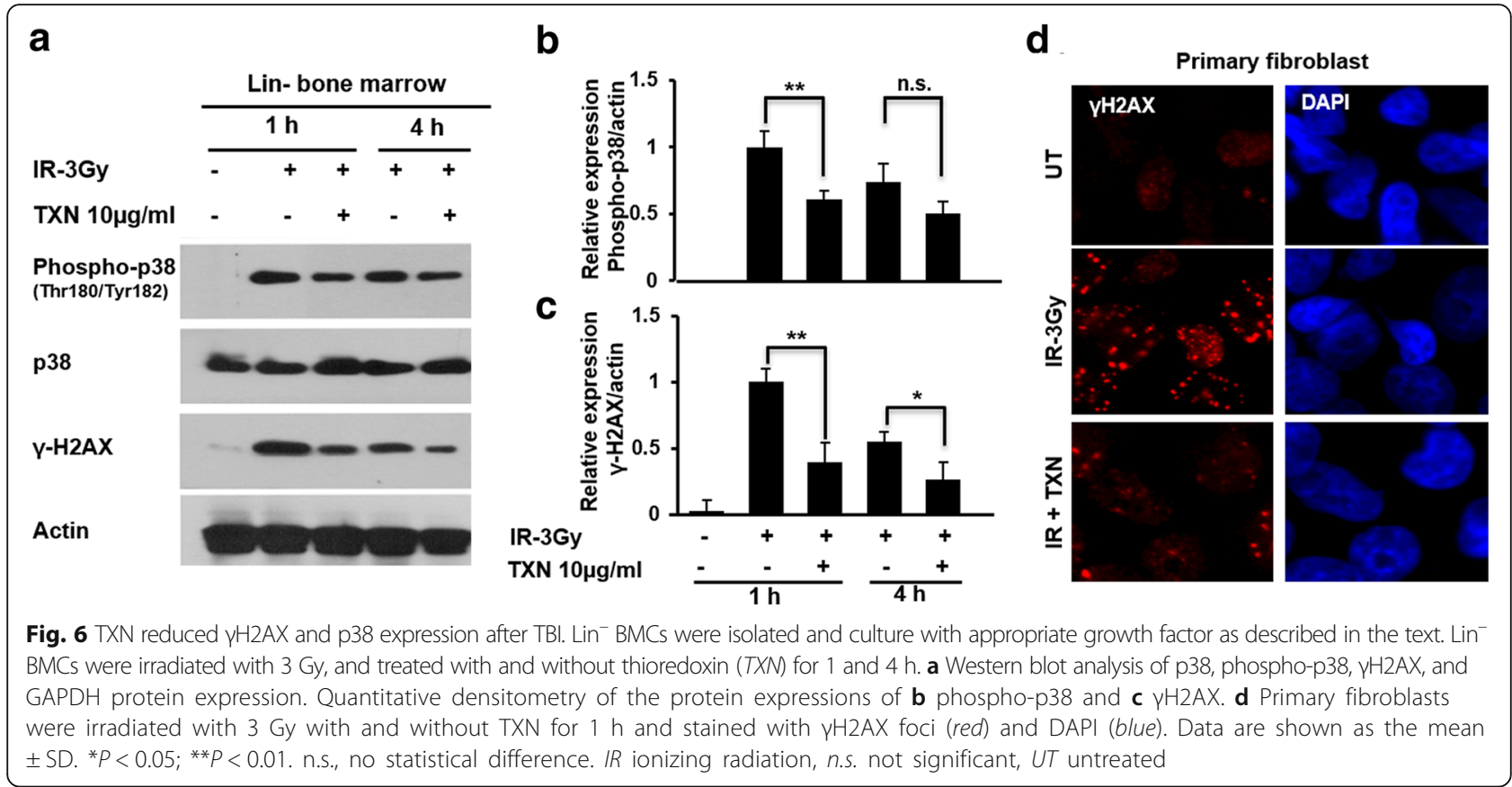

repopulating capacity [44]. Originally described as an antioxidant, TXN also plays numerous roles as a transcription factor and signaling molecule [20,21]. The levels of TXN were found to correlate with organismal lifespan [45, 46]. Pharmacological and genetic inhibition of TXN induced premature senescence in skin fibroblasts and hepatic cancer cells, suggesting a role for TXN in the regulation of cell senescence [47, 48]. Our results demonstrated that TXN-treated mice had lower SA-gal-positive cells compared to saline-treated mice. The effects of TXN in reducing cell senescence after radiation injury were further validated in primary fibroblasts. p16 is one of the vital biomarkers and an important mediator for cell senescence [27, 49]. TXN treatment suppressed the p16 expression level, further supporting the effects of TXN on cell senescence.

Activation of the p38 pathway contributes to the induction of p16 and HSC senescence following exposure to irradiation $[43,50]$. It has been shown that radiation causes HSC cell senescence through the activation of the p38 pathway, and the inhibition of p38 activity with a specific inhibitor (SB203580) attenuated radiation-induced hematopoietic cell injury. Inhibition of p38 activity appears to be a promising strategy for HSC proliferation. We have shown that TXN is able to downregulate phosphorylated p38. TXN serves as a potential mediator of redox signaling by ROS-dependent and -independent pathways $[20,51]$ and is involved in the regulation of multiple biological processes such as antiapoptotic, antiinflammatory, and mitogenic activities [20]. The major target of TXN in the cytosol is apoptosis signal-regulating kinase 1 (ASK1). ASK1 is a member of MAP3 kinase family, which activates both the c-Jun $\mathrm{N}$-terminal kinase (JNK) and p38 MAPK pathways [52]. TXN binds to ASK1 and prevents ASK1 from full activation, thus downregulating the p38 pathway [29].

Radiation-induced DNA damage can occur due to the direct effect of radiation on DNA molecules, which accounts for $30-40 \%$ of lesions, or by free radicals, which accounts for $60-70 \%$ of lesions $[39,53]$. Irradiation induces a variety of DNA lesions, including oxidized base damage, abasic sites, single-strand breaks (SSBs), double-strand breaks (DSBs), and DNA protein crosslinks [54]. DSBs are thought to be the most lethal lesion induced by irradiation, as one unrepaired DSB can be sufficient to trigger apoptosis $[8,17,22] . \gamma \mathrm{H} 2 \mathrm{AX}$ is a vital marker for DSBs. We have found that TXN reduces $\gamma \mathrm{H} 2 \mathrm{AX}$ expression in both murine $\mathrm{Lin}^{-}$bone marrow cells and in primary fibroblasts. The reduction in $\gamma \mathrm{H} 2 \mathrm{AX}$ expression could be due to less double-strand DNA breaks from its antioxidant function and/or enhanced DNA repair by TXN. Our preliminary data indicate that TXN could upregulate the gene expression of the Fanconi anemia/BRCA DNA repair pathway (data not shown).

Radiation damage is complex and there are many mechanisms underlying radiation damage such as iNOS and cytokines [24], miRNA regulation [55], NF-kB activation [56], caspase-dependent apoptosis [8], and LC-II-induced autophagy [57]. In addition to reducing cell senescence and downregulating p38 and $\gamma \mathrm{H} 2 \mathrm{AX}$ as shown in the current study, TXN likely acts on other signaling pathways and affects various cellular events. TXN can act as a cellular growth factor and promotes 
the proliferation of B cells and various transformed cells $[58,59]$. Since ERK $1 / 2$ and JNK are members of the MAPK family, TXN modulation on ERK1/2 and JNK after radiation cannot be excluded. Further studies with these two enzymatic molecules should be explored. Recent studies have implicated TXN in the regulation of cell cycle progression through G2/M [60] and in the p53-mediated base excision repair pathway [61]. TXN activates the MEKK1-JNK signaling pathway, leading to IkB degradation and NF-kB activation [56]. It has been shown that TXN directly interacts with PTEN, inhibits phosphatase activity and membrane binding of PTEN, and activates the Akt pathway [62]. TXN can translocate to the nucleus and regulates the functions of several transcription factors including Ref-1, GR, HSF1, HDAC4, HIF1a, NFkB, Nrf2, PPARg, RUNX2, and SP1 [63, 64].

In the current study, TXN was given intravenously every other day for five doses. We are currently optimizing thioredoxin administration regimens and testing different administration routes, including intramuscular or subcutaneous injection. Intramuscular or subcutaneous injection will offer a simpler and more practical route of administration, particularly in a mass casualty scenario. Importantly, TXN has several important features that make it an attractive candidate for further development as a radiation mitigator. TXN promotes the recovery of hematopoietic stem cells and enhances the recovery of multiple lineages of hematopoietic cells. This is significant as G-CSF only works on myeloid progenitors and only enhances the recovery of neutrophils. TXN affects and modulates diverse cellular events, including cell senescence, apoptosis, and double-strand DNA breaks. TXN is a ubiquitously expressed endogenous protein, eliminating the concerns of developing immune response following administration. TXN can cross the cell membrane and enter cells efficiently. Therefore, TXN can be simply added into an HSC culture or administered systematically.

\section{Conclusion}

In summary, our study shows that TXN effectively mitigates TBI-induced hematopoietic injury in mice, even when given $24 \mathrm{~h}$ after radiation exposure. Our results demonstrate that TXN can be potentially used as an effective medical radiation countermeasure.

\footnotetext{
Abbreviations

ASK1: Apoptosis signal-regulating kinase-1; BMC: Bone marrow cell; BSA: Bovine serum albumin; CFU-GEMM: Colony forming unit-granulocyte, erythroid, macrophage, megakaryocyte; CFU-GM: Colony forming unit-granulocyte macrophage; CFU: Colony forming units; DSB: Double-strand break; FBS: Fetal bovine serum; G-CSF: Granulocyte-colony stimulating factor; H\&E: Hematoxylin and eosin; HGF: Hematopoietic growth factor; HSC: Hematopoietic stem cell; JNK: c-Jun N-terminal kinase; KSL: C-Kit ${ }^{+}$Sca-1 ${ }^{+}$Lin ${ }^{-}$; MAPK: Mitogen-activated protein kinase; PBS: Phosphate-buffered saline; RBC: Red blood cell; ROS: Reactive oxygen species; SA-gal: Senescenceassociated $\beta$-galactosidase; SSB: Single strand break; TBI: Total body irradiation; TXN: Thioredoxin; WBC: White blood cell
}

\section{Acknowledgement}

Not applicable.

\section{Funding}

This work was supported by NHLBI K08HL103780, NCI R01CA197792, and a pilot grant from the Opportunity Funds Management Core of the Centers for Medical Countermeasures against Radiation, National Institute of Allergy and Infectious Diseases; grant number U19A1067773. The funding agents had no roles in the design of the study, in the collection, analysis and interpretation of data, or in writing the manuscript.

\section{Availability of data and materials}

The authors declare that all the data supporting the findings of this study are available within the article and that no data sharing is applicable to this article.

\section{Authors' contributions}

PS analyzed the data and wrote the manuscript; QW performed the experiments; ZZ and YJ assisted with the experiments; BJC, PLD, and NJC provided reagents and input on research design; YK conceived and designed the study, and wrote the manuscript. All authors read and approved the final manuscript.

\section{Ethics approval}

All our studies were performed in accordance with Duke University Institutional Animal Care and Use Committee approved procedures (A096-17-04).

\section{Consent for publication}

Not applicable.

\section{Competing interests}

The authors declare that they have no competing interests.

\section{Publisher's Note}

Springer Nature remains neutral with regard to jurisdictional claims in published maps and institutional affiliations.

\section{Author details}

'Division of Hematological Malignancies and Cellular Therapy, Duke University Medical Center, DUMC 3961, 2400 Pratt Street, Suite 9000, Durham, NC 27710, USA. ${ }^{2}$ Department of Hematology, Fujian Provincial Key Laboratory of Hematology, Fujian Medical University Union Hospital, Fuzhou, Fujian 350001, China.

Received: 9 August 2017 Revised: 3 October 2017

Accepted: 24 October 2017 Published online: 15 November 2017

\section{References}

1. Waselenko JK, MacVittie TJ, Blakely WF, Pesik N, Wiley AL, Dickerson WE, et al. Medical management of the acute radiation syndrome: recommendations of the Strategic National Stockpile Radiation Working Group. Ann Intern Med. 2004;140:1037-51.

2. Dainiak N. Hematologic consequences of exposure to ionizing radiation. Exp Hematol. 2002;30:513-28.

3. Mettler Jr FA, Voelz GL. Major radiation exposure - what to expect and how to respond. N Engl J Med. 2002;346:1554-61.

4. Fendler W, Malachowska B, Meghani K, Konstantinopoulos PA, Guha C, Singh VK, et al. Evolutionarily conserved serum microRNAs predict radiationinduced fatality in nonhuman primates. Sci Transl Med. 2017;9:eaal2408.

5. Greenberger JS. Radioprotection. In Vivo. 2009;23:323-36.

6. Xu G, Wu H, Zhang J, Li D, Wang Y, Wang Y, et al. Metformin ameliorates ionizing irradiation-induced long-term hematopoietic stem cell injury in mice. Free Radic Biol Med. 2015:87:15-25.

7. Wang Y, Schulte BA, LaRue AC, Ogawa M, Zhou D. Total body irradiation selectively induces murine hematopoietic stem cell senescence. Blood. 2006;107:358-66.

8. Shao L, Luo Y, Zhou D. Hematopoietic stem cell injury induced by ionizing radiation. Antioxid Redox Signal. 2014;20:1447-62.

9. Greenberger JS, Epperly MW. Antioxidant therapeutic approaches toward amelioration of the pulmonary pathophysiological damaging effects of ionizing irradiation. Curr Respiratory Med Rev. 2007;3:29-37. 
10. Singh VK, Romaine PL, Seed TM. Medical countermeasures for radiation exposure and related injuries: characterization of medicines, FDA-approval status and inclusion into the strategic national stockpile. Health Phys. 2015;108:607.

11. Singh VK, Newman VL, Seed TM. Colony-stimulating factors for the treatment of the hematopoietic component of the acute radiation syndrome (H-ARS): a review. Cytokine. 2015;71:22-37.

12. Peslak SA, Wenger J, Bemis JC, Kingsley PD, Koniski AD, McGrath KE, Palis J. EPO-mediated expansion of late-stage erythroid progenitors in the bone marrow initiates recovery from sublethal radiation stress. Blood. 2012;120:2501-11.

13. Zhou D, Deoliveira D, Kang Y, Choi SS, Li Z, Chao NJ, et al. Insulin-like growth factor 1 mitigates hematopoietic toxicity after lethal total body irradiation. Int J Radiat Oncol Biol Phys. 2013;85:1141-8.

14. Spitzer G, Adkins DR, Spencer V, Dunphy FR, Petruska PJ, Velasquez WS, et al. Randomized study of growth factors post-peripheral-blood stem-cell transplant: neutrophil recovery is improved with modest clinical benefit. J Clin Oncol. 1994;12:661-70.

15. Reeves G. Overview of use of G-CSF and GM-CSF in the treatment of acute radiation injury. Health Phys. 2014;106:699-703.

16. Dainiak N, Gent RN, Carr Z, Schneider R, Bader J, Buglova E, et al. First global consensus for evidence-based management of the hematopoietic syndrome resulting from exposure to ionizing radiation. Disaster Med Public Health Prep. 2011;5:202-12.

17. Wagemaker G, Neelis K, Hartong SC, Wognum AW, Thomas GR, Fielder PJ, et al. The efficacy of recombinant TPO in murine and nonhuman primate models for myelosuppression and stem cell transplantation. Stem Cells. 1998;16:127-41.

18. An N, Janech M, Bland A, Lazarchick J, Arthur JM, Kang Y. Proteomic analysis of murine bone marrow niche microenvironment identifies thioredoxin as a novel agent for radioprotection and for enhancing donor cell reconstitution. Exp Hematol. 2013;41:944-56.

19. Collet JF, Messens J. Structure, function, and mechanism of thioredoxin proteins. Antioxid Redox Signal. 2010;13:1205-16.

20. An N, Kang Y. Thioredoxin and hematological malignancies. In: Townsend Tew K, editor. Redox and cancer: advances in cancer research. 2014.

21. Lee S, Kim SM, Lee RT. Thioredoxin and thioredoxin target proteins: from molecular mechanisms to functional significance. Antioxid Redox Signal. 2013;18:1165-207.

22. Chen BJ, Deoliveira D, Spasojevic I, Sempowski GD, Jiang C, Owzar K, et al. Growth hormone mitigates against lethal irradiation and enhances hematologic and immune recovery in mice and nonhuman primates. PLoS One. 2010;5:e11056.

23. Venkateswaran K, Shrivastava A, Agrawala PK, Prasad A, Kalra N, Pandey PR, et al. Mitigation of radiation-induced hematopoietic injury by the polyphenolic acetate 7, 8-diacetoxy-4-methylthiocoumarin in mice. Sci Rep. 2016;6:37305.

24. Kiang JG, Jiao W, Cary L, Mog S, Elliott T, Pellmar T, et al. Wound trauma increases radiation-induced mortality by increasing iNOS, cytokine concentrations, and bacterial infections. Radiat Res. 2010;173(3):319-32.

25. Meng A, Wang $Y$, Van Zant G, Zhou D. lonizing radiation and busulfan induce premature senescence in murine bone marrow hematopoietic cells. Cancer Res. 2003:63:5414-9.

26. Cho J, Shen $H, Y u$ H, Li H, Cheng T, Lee SB, et al. Ewing sarcoma gene Ews regulates hematopoietic stem cell senescence. Blood. 2011;117:1156-66.

27. Hilpert M, Legrand C, Bluteau D, Balayn N, Betems A, Bluteau O, et al. p19 INK4d controls hematopoietic stem cells in a cell-autonomous manner during genotoxic stress and through the microenvironment during aging. Stem Cell Rep. 2014;3:1085-102.

28. Kiang JG, Garrison BR, Smith JT, Fukumoto R. Ciprofloxacin as a potential radio-sensitizer to tumor cells and a radio-protectant for normal cells: differential effects on $\mathrm{Y}$-H2AX formation, p53 phosphorylation, $\mathrm{BCl}-2$ production, and cell death. Mol Cell Biochem. 2014;393:133-43.

29. Kiang JG, Fukumoto R. Ciprofloxacin increases survival after ionizing irradiation combined injury: $\mathrm{Y}-\mathrm{H} 2 \mathrm{AX}$ formation, cytokine/chemokine, and red blood cells. Health Phys. 2014;106:720-26.

30. Mariotti LG, Pirovano G, Savage Kl, Ghita M, Ottolenghi A, Prise KM, et al. Use of the gamma-H2AX assay to investigate DNA repair dynamics following multiple radiation exposures. PLoS One. 2013;8:e79541.

31. Kuo LJ, Yang L-X. Y-H2AX-a novel biomarker for DNA double-strand breaks. In vivo. 2008;22:305-9.

32. Coleman CN, Stone HB, Moulder JE, Pellmar TC. Modulation of radiation injury. Science. 2004;304:693-4.

33. Xue X-L, Han X-D, Li Y, Chu X-F, Miao W-M, Zhang J-L, et al. Astaxanthin attenuates total body irradiation-induced hematopoietic system injury in mice via inhibition of oxidative stress and apoptosis. Stem Cell Res Ther. 2017;8:7.

34. Zhang H, Zhai Z, Wang Y, Zhang J, Wu H, Wang Y, et al. Resveratrol ameliorates ionizing irradiation-induced long-term hematopoietic stem cell injury in mice. Free Radic Biol Med. 2013;54:40-50.

35. Mouthon MA, Van der Meeren A, Gaugler MH, Visser TP, Squiban C et al. Thrombopoietin promotes hematopoietic recovery and survival after high-dose whole body irradiation. Int J Radiat Oncol Biol Phys. 1999;43:867-75.

36. Yalcin S, Zhang X, Luciano JP, Mungamuri SK, Marinkovic D, Vercherat C, et al. Foxo3 is essential for the regulation of ataxia telangiectasia mutated and oxidative stress-mediated homeostasis of hematopoietic stem cells. J Biol Chem. 2008;283:25692-705.

37. Tothova Z, Kollipara R, Huntly BJ, Lee BH, Castrillon DH, Cullen DE, et al. FoxOs are critical mediators of hematopoietic stem cell resistance to physiologic oxidative stress. Cell. 2007;128:325-39.

38. Ward JF, Evans JW, Limoli CL, Calabro-Jones PM. Radiation and hydrogen peroxide induced free radical damage to DNA. Br J Cancer Suppl. 1987;8:105-12.

39. Santivasi WL, Xia F. lonizing radiation-induced DNA damage, response, and repair. Antioxid Redox Signal. 2014;21:251-9.

40. Meng A, Wang Y, Brown SA, Van Zant G, Zhou D. lonizing radiation and busulfan inhibit murine bone marrow cell hematopoietic function via apoptosis-dependent and -independent mechanisms. Exp Hematol. 2003;31:1348-56.

41. Neben S, Hellman S, Montgomery M, Ferrara J, Mauch P. Hematopoietic stem cell deficit of transplanted bone marrow previously exposed to cytotoxic agents. Exp Hematol. 1993;21:156-62.

42. van Os R, Robinson S, Sheridan T, Mislow JM, Dawes D, Mauch PM. Granulocyte colony-stimulating factor enhances bone marrow stem cell damage caused by repeated administration of cytotoxic agents. Blood. 1998:92:1950-6.

43. Wang Y, Liu L, Zhou D. Inhibition of p38 MAPK attenuates ionizing radiation-induced hematopoietic cell senescence and residual bone marrow injury. Radiat Res. 2011;176:743-52.

44. Hellman S, Botnick LE. Stem cell depletion: an explanation of the late effects of cytotoxins. Int J Radiat Oncol Biol Phys. 1977;2:181-4.

45. Mitsui A, Hamuro J, Nakamura H, Kondo N, Hirabayashi Y, Ishizaki-Koizumi S, et al. Overexpression of human thioredoxin in transgenic mice controls oxidative stress and life span. Antioxid Redox Signal. 2002;4:693-6.

46. Miranda-Vizuete A, González JCF, Gahmon G, Burghoorn J, Navas P, Swoboda P. Lifespan decrease in a Caenorhabditis elegans mutant lacking TRX-1, a thioredoxin expressed in ASJ sensory neurons. FEBS Lett. 2006:580:484-90

47. Young JJ, Patel A, Rai P. Suppression of thioredoxin-1 induces premature senescence in normal human fibroblasts. Biochem Biophys Res Commun. 2010:392:363-8.

48. Zhang T, Liu H, Zhu C, Briggs K, Ya K, Fleming JA, et al. Silencing thioredoxin induces liver cancer cell senescence under hypoxia. Hepatol Res. 2012:42:706-13.

49. Liu Y, Sanoff HK, Cho H, Burd CE, Torrice C, Ibrahim JG, et al. Expression of p16INK4a in peripheral blood T-cells is a biomarker of human aging. Aging Cell. 2009;8:439-48.

50. Ito K, Hirao A, Arai F, Takubo K, Matsuoka S, Miyamoto K, et al. Reactive oxygen species act through p38 MAPK to limit the lifespan of hematopoietic stem cells. Nat Med. 2006;12:446-51.

51. Antman KS, Griffin JD, Elias A, Socinski MA, Ryan L, Cannistra SA, et al. Effect of recombinant human granulocyte-macrophage colonystimulating factor on chemotherapy-induced myelosuppression. N Engl J Med. 1988;319:593-8.

52. Ichijo $H$, Nishida E, Irie $K$, ten Dijke $P$, Saitoh $M$, Moriguchi $T$, et al. Induction of apoptosis by ASK1, a mammalian MAPKKK that activates SAPK/JNK and p38 signaling pathways. Science. 1997;275:90-4.

53. Ward J. DNA damage produced by ionizing radiation in mammalian cells: identities, mechanisms of formation, and reparability. Prog Nucleic Acid Res Mol Biol. 1988;35:95-125.

54. Bucher N, Britten C. G2 checkpoint abrogation and checkpoint kinase-1 targeting in the treatment of cancer. Br J Cancer. 2008;98:523-8.

55. Chaudhry MA. Radiation-induced microRNA: discovery, functional analysis, and cancer radiotherapy. J Cell Biochem. 2014:115:436-49.

56. Das KC. C-Jun NH2-terminal kinase-mediated redox-dependent degradation of $\mid \mathrm{KB}$ : role of thioredoxin in NF-KB activation. J Biol Chem. $2001 ; 276: 4662-70$ 
57. Hou J, Han Z, Jing Y, Yang X, Zhang S, Sun K, et al. Autophagy prevents irradiation injury and maintains stemness through decreasing ROS generation in mesenchymal stem cells. Cell Death Dis. 2013;4:e844.

58. Powis $G$, Mustacich $D$, Coon $A$. The role of the redox protein thioredoxin in cell growth and cancer. Free Radic Biol Med. 2000;29:312-22.

59. Blum H, Röllinghoff $M$, Gessner A. Expression and co-cytokine function of murine thioredoxin/adult T cell leukaemia-derived factor (ADF). Cytokine. 1996:8:6-13.

60. Moon M, Kim J, Kim T, Yum J, Cho E, Kim I. Polyamine depletion partially reduces the radiation-induced cell death via cell cycle delay mediated by thioredoxin. Cell Biol Toxicol. 2006;22:137-47.

61. Kim HL, Koedrith P, Lee SM, Kim YJ, Seo YR. Base excision DNA repair defect in thioredoxin-1 (Trx1)-deficient cells. Mutat Res. 2013;751:1-7.

62. Song Z, Saghafi N, Gokhale V, Brabant M, Meuillet EJ. Regulation of the activity of the tumor suppressor PTEN by thioredoxin in Drosophila melanogaster. Exp Cell Res. 2007;313:1161-71.

63. Ago T, Liu T, Zhai P, Chen W, Li H, Molkentin JD, et al. A redox-dependent pathway for regulating class II HDACs and cardiac hypertrophy. Cell. 2008; 133:978-93.

64. Matthews JR, Wakasugi N, Yodoi J, Hay RT. Thioredoxin regulates the DNA binding activity of NF-kB by reduction of a disulphide bond involving cysteine 62. Nucleic Acids Res. 1992;20:3821-30.

Submit your next manuscript to BioMed Central and we will help you at every step:

- We accept pre-submission inquiries

- Our selector tool helps you to find the most relevant journal

- We provide round the clock customer support

- Convenient online submission

- Thorough peer review

- Inclusion in PubMed and all major indexing services

- Maximum visibility for your research

Submit your manuscript at www.biomedcentral.com/submit
Biomed Central 